\title{
Ge-on-Si based mid-infrared plasmonics
}

Frigerio, Jacopo, Baldassarre, Leonetta, Pellegrini, Giovanni, Fischer, Marco, Gallacher, Kevin, et al.

Jacopo Frigerio, Leonetta Baldassarre, Giovanni Pellegrini, Marco P. Fischer, Kevin Gallacher, Ross Millar, Andrea Ballabio, Daniele Brida, Giovanni Isella, Enrico Napolitani, Douglas J. Paul, Michele Ortolani, Paolo Biagioni, "Ge-onSi based mid-infrared plasmonics," Proc. SPIE 11691, Silicon Photonics XVI, 116910M (5 March 2021); doi: 10.1117/12.2576175

SPIE. Event: SPIE OPTO, 2021, Online Only 


\title{
Ge-on-Si based mid-infrared plasmonics
}

\author{
Jacopo Frigerio*a, Leonetta Baldassarre ${ }^{\mathrm{b}}$, Giovanni Pellegrini ${ }^{\mathrm{c}}$, Marco P. Fischer ${ }^{\mathrm{d}}$, Kevin Gallacher \\ ${ }^{\mathrm{e}}$, Ross Millar ${ }^{\mathrm{e}}$, Andrea Ballabio ${ }^{\mathrm{a}}$, Daniele Brida ${ }^{\mathrm{f}}$, Giovanni Isella ${ }^{\mathrm{a}}$, Enrico Napolitani ${ }^{\mathrm{g}}$, Douglas J. \\ Paul ${ }^{\mathrm{e}}$, Michele Ortolani ${ }^{\mathrm{b}}$, Paolo Biagioni ${ }^{\mathrm{c}}$ \\ ${ }^{a}$ L-NESS, Dip. Di Fisica, Politecnico di Milano, Via Anzani 42, I-22100 Como, Italy; \\ ${ }^{\mathrm{b}}$ Dip. Di Fisica, Sapienza Università di Roma, Piazzale Aldo Moro 5, I-00185 Rome, Italy; \\ ${ }^{c}$ Dip. Di Fisica, Politecnico di Milano, Piazza Leonardo da Vinci 32, I-20133, Milano, Italy; \\ ${ }^{\mathrm{d}}$ Dep. Of Physics and Center for App. Photonics, University of Konstanz, D-78457 Konstanz, \\ Germany. \\ e School of Engineering, University of Glasgow, Rankine Building, Oakfield avenue, Glasgow, \\ G128LT, United Kingdom \\ ${ }^{\mathrm{f}}$ Faculté des Sciences, de la Technologie et de la Communication, Université de Luxembourg, L- \\ 1511 Luxembourg \\ g Dipartimento di Fisica e Astronomia, Università di Padova and CNR-IMM MATIS, Via Marzolo \\ 8, I-35131 Padova, Italy
}

\begin{abstract}
In the last decade, silicon photonics has undergone an impressive development driven by an increasing number of technological applications. Plasmonics has not yet made its way to the microelectronic industry, mostly because of the lack of compatibility of typical plasmonic materials with foundry processes. In this framework, we have developed a plasmonic platform based on heavily $\mathrm{n}$-doped Ge grown on silicon substrates. We developed growth protocols to reach $\mathrm{n}$ doping levels exceeding $10^{20} \mathrm{~cm}^{-3}$, allowing us to tune the plasma wavelength of $\mathrm{Ge}$ in the 3-15 $\mu \mathrm{m}$ range. The plasmonic resonances of Ge-on-Si nanoantennas have been predicted by simulations, confirmed by experimental spectra and exploited for molecular sensing. Our work represents a benchmark for group-IV mid-IR plasmonics.
\end{abstract}

Keywords: Germanium, Plasmonics, Mid-infrared, Sensing, Doping

\section{INTRODUCTION}

Mid-infrared (MIR) photonics is receiving a remarkable attention due to the envisaged applications in biochemistry [1], chemical analytics [2], environmental monitoring for safety and security [3] and healthcare [4]. The interest towards the MIR resides in the presence of the vibrational and rotational absorption lines of molecules in the wavelength range $\lambda=4$ to $15 \mu \mathrm{m}$. The possibility to directly probe vibrational lines via optical absorption [5] allows for a high degree of molecular sensitivity, with countless applications including e.g. the determination of the conformational state of proteins, which constitutes the key toward an early diagnose of neurodegenerative diseases and cancer. In this context, many research efforts have been devoted to find conducting thin films displaying values of the complex dielectric function $\varepsilon(\omega)=$ $\varepsilon^{\prime}(\omega)+i \varepsilon^{\prime \prime}(\omega)$ that can be tailored to meet the needs of novel electromagnetic designs exploiting the concepts of metamaterials, transformation optics and plasmonics [6]. Such a requirement is met by elemental metal at visible and near-infrared wavelengths, but the same condition cannot be achieved in the MIR range because they possess an extremely high negative value of $\varepsilon^{\prime}$ not equalled, in absolute value on the positive side, by any dielectric material. It has been proposed that, in order to obtain tunable values of $\varepsilon^{\prime}$ over the entire MIR range [7,8], heavily doped semiconductors [9-15] may be used because their free carrier density can be set by selecting the doping level and further tuned by electrostatic gating [12] or optical excitation [16-18]. In recent years group-IV [10,11,15,19] and compound III-V [14,17] semiconductors have been extensively investigated for MIR plasmonics [20]. Among them, Ge is considered particularly interesting because it features a plasma frequency easily tunable between 3-15 $\mu \mathrm{m}$ by electron-doping and because it can be directly deposited on Si substrates.

*jacopo.frigerio@polimi.it

Silicon Photonics XVI, edited by Graham T. Reed, Andrew P. Knights, Proc. of SPIE Vol. 11691, $116910 \mathrm{M} \cdot$ ? $2021 \mathrm{SPIE} \cdot \mathrm{CCC}$ code: $0277-786 \mathrm{X} / 21 / \$ 21 \cdot$ doi: $10.1117 / 12.2576175$ 
In addition, Ge-on-Si [21-24] and SiGe-on-Si [25-32] MIR photonic platforms are currently under development, foreshadowing the merging of plasmonic and photonic functionalities on the same chip. The high-frequency limit for the conducting behavior of a given material with free carrier density $n$ and conductivity effective mass $\mathrm{m}^{*}$ is ultimately set by the screened plasma frequency or the frequency $\omega^{*}$ such that $\varepsilon^{\prime}\left(\omega^{*}\right)=0$, approximately given (in the limit of low losses) by:

$$
\omega^{*} \cong \sqrt{\frac{n e^{2}}{\varepsilon_{0} \varepsilon_{\infty} m^{*}}}
$$

where $\varepsilon_{\infty}$ is the high frequency dielectric constant and e is the electron charge. This work is devoted to a study of IR plasmonics in $\mathrm{n}$-doped germanium ( $\mathrm{n}-\mathrm{Ge}$ ) thin films, which display ideal intrinsic properties including $\mathrm{n}$ spanning two orders of magnitude, a relatively small $\mathrm{m}^{*}=0.12 \mathrm{me}$ and $\omega *$ reaching values well into the mid-IR.

\section{GROWTH OF HEAVILY N-DOPED GERMANIUM EPILAYERS}

All the samples presented in this work have been grown by low-energy plasma-enhanced chemical vapour deposition (LEPECVD) [33]. A schematics of the growth reactor is shown in Fig 1. The technique relies on the use of a low energy plasma in order to enhance the deposition efficiency with respect to conventional chemical vapor deposition (CVD). In a LEPECVD reactor, a tantalum filament inside the plasma source is heated by a high current $(130 \mathrm{~A})$, so that electrons are thermionically emitted. Argon gas is passed up through the plasma source into the growth chamber. A direct current arc discharge of 20-80 A with a low voltage of $30 \mathrm{~V}$ can then be sustained between the filament and the growth chamber; an anode ring is mounted within the growth chamber to stabilize the discharge. Magnetic fields (created by coils) are used to focus the plasma onto the substrate, which is heated from behind by a graphite heater. The deposition chamber has a base pressure of $10^{-9} \mathrm{mbar}$, while the working pressure is much higher reaching $10^{-2}$ mbar. Precursors gases $\left(\mathrm{GeH}_{4}\right.$ and $\mathrm{SiH}_{4}$ for germanium and silicon, $\mathrm{PH}_{3}$ and $\mathrm{B}_{2} \mathrm{H}_{6}$ for doping) are introduced in the chamber. The gas flows are regulated by mass flow controllers with a maximum flux of $25 \mathrm{sccm}$ for $\mathrm{GeH}_{4}$ and $\mathrm{SiH}_{4}$ and of $10 \mathrm{sccm}$ for $\mathrm{B}_{2} \mathrm{H}_{6}$ and $\mathrm{PH}_{3} . \mathrm{B}_{2} \mathrm{H}_{6}$ and $\mathrm{PH} 3$ are diluited in Ar respectively at $1 \%$ and $5 \%$. The growth rate, controlled by the plasma density and by the amount of process gas, and the mobility of the adatoms, controlled by the substrate temperature, can be optimized separately, giving to LEPECVD a great flexibility for the growth of high quality SiGe thin films.

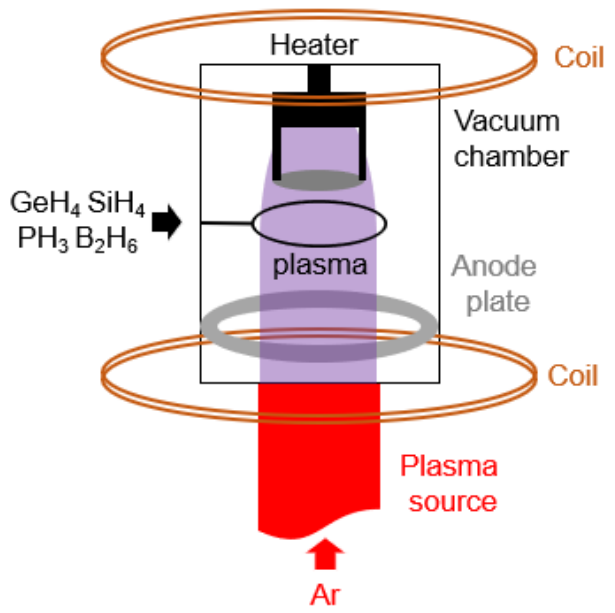

Figure 1: Schematics of a LEPECVD reactor

The main goal to be achieved in terms of material properties consists in obtaining Ge-on-Si epilayers featuring an electron density in the $10^{17}-10^{20} \mathrm{~cm}^{-3}$ range, which translates in a plasma wavelength tuning range spanning the full MIR between 3-15 $\mu \mathrm{m}$ according to eq. 1 . In order to achieve this goal, we have carefully measured the active and incorporated doping 
as a function of different growth parameters such as the $\mathrm{PH}_{3} / \mathrm{GeH}_{4}$ flux ratio, the growth temperature and the density of threading dislocations. The active doping concentration of all the samples were determined by four point Hall effect measurements performed at room temperature on six-terminals Hall bar structures. The Hall bars were patterned by standard UV lithography and dry etching. A metal stack composed by $\mathrm{Ni}(100 \mathrm{~nm}) / \mathrm{Ti}(5 \mathrm{~nm}) / \mathrm{Al}(100 \mathrm{~nm})$ was deposited by an e-beam evaporator on the measurement pads of the Hall bars and then annealed at $340{ }^{\circ} \mathrm{C}$ for $30 \mathrm{~s}$ in a rapid thermal annealer (RTA) in order to realize ohmic contacts. A magnetic field of $0.2 \mathrm{~T}$ was applied in the direction perpendicular to the sample plane by a permanent magnet to perform Hall effect measurements. The density of incorporated $\mathrm{P}$ atoms has been measured by secondary ion mass spectroscopy (SIMS). The reflectivity as a function of the wavenumber has been measured by Fourier Transform Infrared Spectroscopy (FTIR).

\subsection{Active doping as a function of the $\mathrm{PH}_{3} / \mathrm{GeH}_{4}$ flux ratio}

A first series of samples has been realized in order to understand the main factors limiting the maximum doping level achievable in standard growth conditions. All the samples consist in a $500 \mathrm{~nm} \mathrm{Ge}$ epilayer grown at $500{ }^{\circ} \mathrm{C}$ and at a rate of $1 \mathrm{~nm} / \mathrm{s}$ on an intrinsic $\mathrm{Si}$ substrate with a resistivity $\rho>10 \mathrm{k} \Omega$. The $\mathrm{GeH}_{4}$ flux has been kept fixed at $20 \mathrm{sccm}$, while the $\mathrm{PH}_{3}$ has been changed for every sample. The main characteristics of the samples are summarized in tab 1.

Table 1. Main characteristics of the first series of samples.

\begin{tabular}{|c|c|c|c|}
\hline Sample & $\begin{array}{c}\mathbf{P H}_{\mathbf{3}} \backslash \mathbf{G e H}_{\mathbf{4}} \\
\text { flux ratio }\end{array}$ & $\begin{array}{c}\text { Active doping } \\
\mathbf{( c m}^{-\mathbf{3}} \mathbf{)}\end{array}$ & $\begin{array}{c}\text { Incorporated } \\
\mathbf{P} \text { atoms } \\
\mathbf{( c m}^{-\mathbf{3}} \mathbf{)}\end{array}$ \\
\hline 8643 & 0.0025 & $3.4 \mathrm{E}+18$ & $3.6 \mathrm{E}+18$ \\
\hline 8644 & 0.0125 & $1.5 \mathrm{E}+19$ & $2.1 \mathrm{E}+19$ \\
\hline 8645 & 0.025 & $1.2 \mathrm{E}+19$ & $2.5 \mathrm{E}+19$ \\
\hline 8648 & 0.00025 & $1.2 \mathrm{E}+17$ & $2.0 \mathrm{E}+17$ \\
\hline 8649 & 0.0063 & $1.0 \mathrm{E}+19$ & $1.3 \mathrm{E}+19$ \\
\hline
\end{tabular}

The active and incorporated doping for the first series of samples is reported in Fig 2a. The P atoms incorporated into the Ge thin film increases for increasing $\mathrm{PH}_{3} / \mathrm{GeH}_{4}$ flux ratios, but this does not hold true for the active doping level, which clearly tends to saturate at $\mathrm{N}_{\mathrm{D}} \approx 1.2 \times 10^{19} \mathrm{~cm}^{-3}$. This phenomenon, which has been recently studied in Refs. 34-35, is due to the formation of vacancy-donor complexes that deactivate most of the donor atoms in the material.

(a)

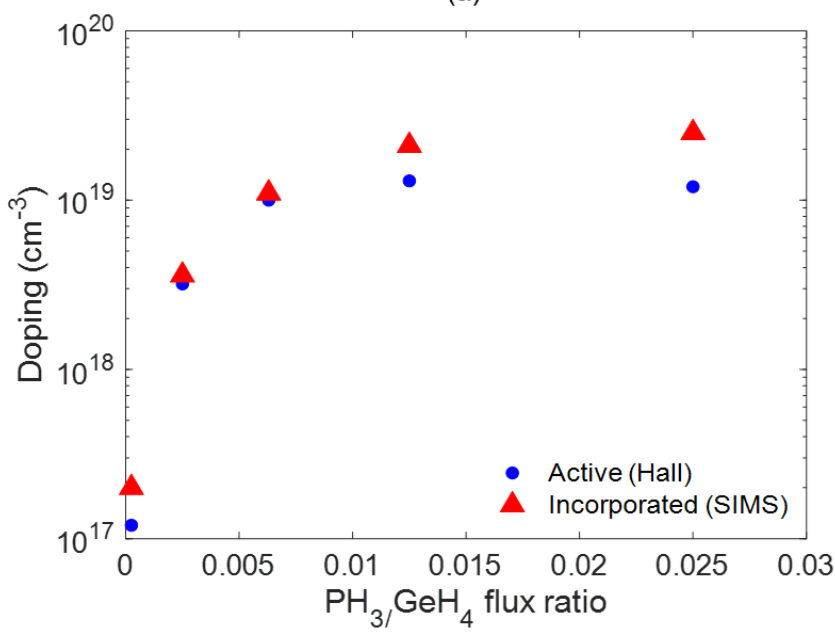

(b)

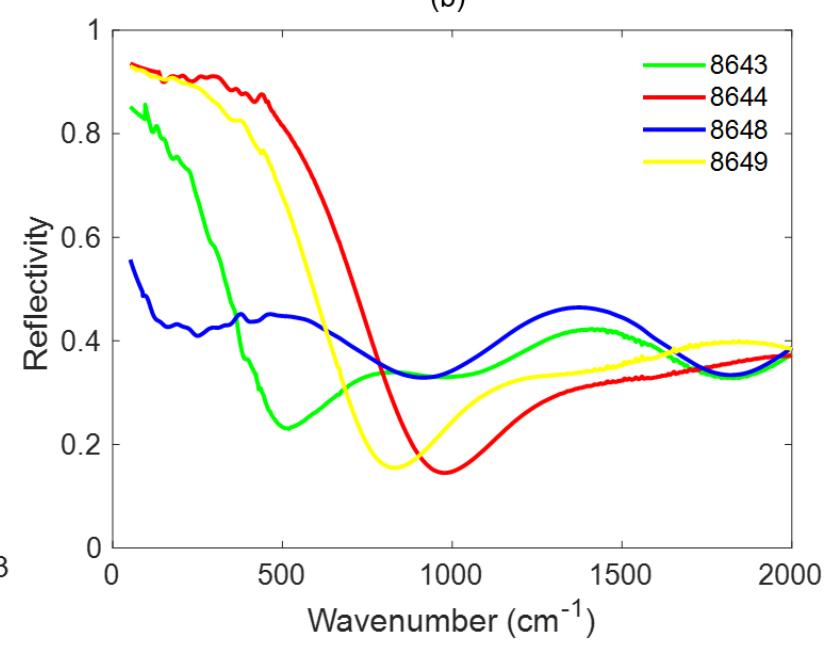

Figure 2: (a) Active (blue dots) and incorporated (red triangles) doping concentration as a function of the $\mathrm{PH}_{3} / \mathrm{GeH}_{4}$ flux ratio; (b) Reflectivity MIR spectra obtained by FTIR spectroscopy. 
The plasma edge (highlighted by the sudden drop in reflectivity associated with the crossing of the screened plasma frequency) for the sample featuring the highest active doping (8644) falls approximately at $1000 \mathrm{~cm}^{-1}(\lambda=10 \mu \mathrm{m}$, see fig. $2 b)$.

\subsection{Influence of the growth temperature on the doping level}

The second series of samples has been grown to investigate the dependence of the doping level as a function of the growth temperature. The samples $9738-9746$ consist in a $500 \mathrm{~nm}$ Ge epilayer grown at a rate of $1 \mathrm{~nm} / \mathrm{s}$ on an intrinsic Si substrate with a resistivity $\rho>10 \mathrm{k} \Omega$. The sample 9854 consists in $100 \mathrm{~nm}$ of Ge grown at a very low rate of $0.1 \mathrm{~nm} / \mathrm{s}$. The $\mathrm{PH}_{3} / \mathrm{GeH}_{4}$ flux ratio has been kept fixed at 0.035 for all the samples. The main features of these samples are summarized in table 2 .

Table 2. Main characteristics of the second series of samples.

\begin{tabular}{|c|c|c|c|}
\hline Sample & $\begin{array}{c}\text { Growth Temp. } \\
\left({ }^{\circ} \mathbf{C}\right)\end{array}$ & $\begin{array}{c}\text { Active doping } \\
\left(\mathbf{c m}^{-3}\right)\end{array}$ & $\begin{array}{c}\text { Incorporated } \\
\mathbf{P ~ a t o m s ~}_{\left(\mathbf{c m}^{-3} \mathbf{)}\right.}\end{array}$ \\
\hline 9738 & 450 & $2.0 \mathrm{E} 19$ & $1.2 \mathrm{E}+20$ \\
\hline 9739 & 460 & $2.3 \mathrm{E}+19$ & $1.0 \mathrm{E}+20$ \\
\hline 9740 & 470 & $2.0 \mathrm{E}+19$ & $7.0 \mathrm{E}+19$ \\
\hline 9743 & 500 & $1.0 \mathrm{E}+19$ & $3.0 \mathrm{E}+19$ \\
\hline 9746 & 530 & $5.0 \mathrm{E}+18$ & $1.0 \mathrm{E} 19$ \\
\hline 9854 & 350 & $1.0 \mathrm{E}+18$ & $2.5 \mathrm{E} 20$ \\
\hline
\end{tabular}

The data reported in figure 3 show a clear increase of the incorporated donor atoms for decreasing temperature, which is due the higher solubility of $\mathrm{P}$ atoms in $\mathrm{Ge}$ at lower temperatures. The trend of the active doping shows a global maximum at $\mathrm{T}=460^{\circ} \mathrm{C}$.

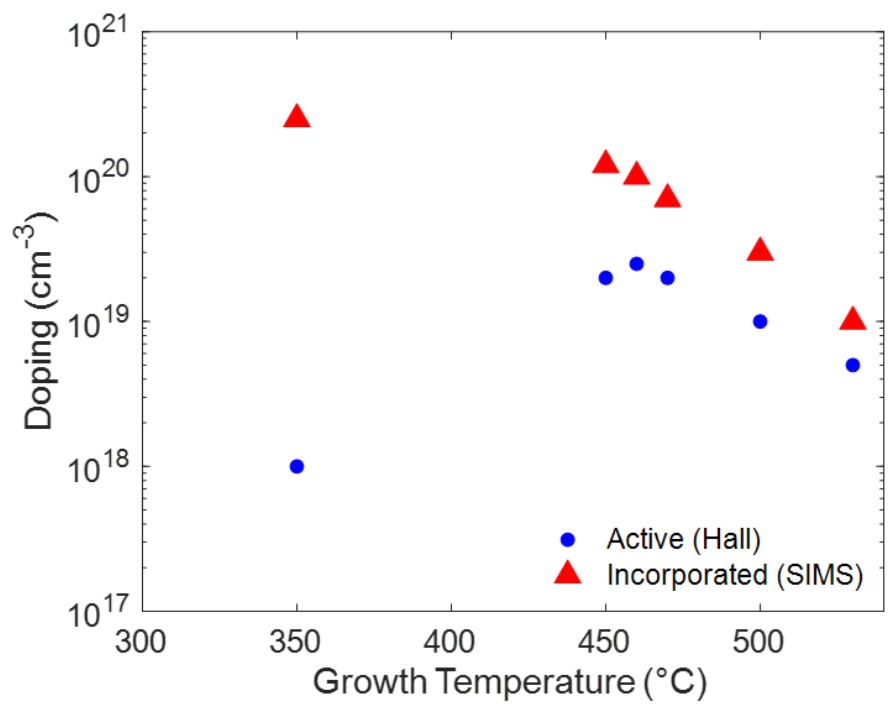

Figure 3: Active (blue dots) and incorporated (red triangles) doping concentration as a function of the growth temperature. 


\subsection{Influence of threading dislocations density on the doping levels.}

One of the main drawbacks of Ge-on-Si epitaxy is the $4.2 \%$ mismatch between the lattice parameters of the two materials, which causes the introduction of defects (misfit and threading dislocations) in the Ge epilayer. Such defects introduce ptype acceptor sites in the Ge thin film [36], thus potentially playing a role in the donor deactivation. Normally the threading dislocation density (TDD) can be reduced by performing thermal annealing cycles after the growth, but such technique cannot be straightforwardly applied to heavily n-doped Ge epilayers. Indeed it is known that such thermal treatments promote the formation of $\mathrm{P}$ clusters, preferentially along the TDs, leading to a poor material quality and strongly nonuniform doping profiles [37]. Three samples featuring different TDD spanning four order of magnitudes have been grown to understand the role played by the TDs onto the deactivation of $\mathrm{P}$ atoms. The sample 9338 consists in a $2 \mu \mathrm{m}$ thick ndoped Ge thin film directly grown on silicon without any thermal treatment. The Ge film has been deposited at $460{ }^{\circ} \mathrm{C}$ at a rate of $1 \mathrm{~nm} / \mathrm{s}$, with a $\mathrm{PH}_{3} / \mathrm{GeH}_{4}$ ratio of 0.035 . This sample features a TDD of $\approx 10^{9} \mathrm{~cm}^{-2}$, similar to the samples discussed in the previous sections.. The sample 9335 consists in a $2 \mu \mathrm{m}$ thick undoped Ge buffer layer grown on intrinsic silicon. The buffer layer has been treated in-situ with six thermal annealing cycles between 600 and $800{ }^{\circ} \mathrm{C}$, thus reaching a TDD of $\approx 10^{7} \mathrm{~cm}^{-2}$. The buffer layer is then covered with a $2 \mu \mathrm{m}$ thick $\mathrm{n}$-doped Ge layer grown under the same conditions as sample 9335 . The sample 9336 consists in a copy of 9335 grown on an intrinsic Ge substrate. Such sample features a TDD of $10^{5} \mathrm{~cm}^{-2}$. SIMS measurements performed on similar samples indicate that the $\mathrm{P}$ incorporation is $\approx 7 \times 10^{19} \mathrm{~cm}^{-3}$ for all the samples. The epitaxial schemes of the samples, together with a plot of the active doping level as a function of the TDD is reported in Fig. 4. The main characteristics of the samples are summarized in table 3.

Table 3. Main characteristics of the third series of samples.

\begin{tabular}{|c|c|c|c|}
\hline Sample & Substrate & $\begin{array}{c}\text { TDD } \\
\left(\mathbf{c m}^{-2}\right)\end{array}$ & $\begin{array}{c}\text { Active doping } \\
\left(\mathbf{c m}^{-3}\right)\end{array}$ \\
\hline 9338 & $\mathrm{Si}$ & $1 \mathrm{E}+9$ & $2.3 \mathrm{E}+19$ \\
\hline 9335 & $\mathrm{Si}+\mathrm{Ge}$ buffer & $1 \mathrm{E}+7$ & $2.5 \mathrm{E}+19$ \\
\hline 9336 & $\mathrm{Ge}$ & $1 \mathrm{E}+5$ & $3.3 \mathrm{E}+19$ \\
\hline
\end{tabular}

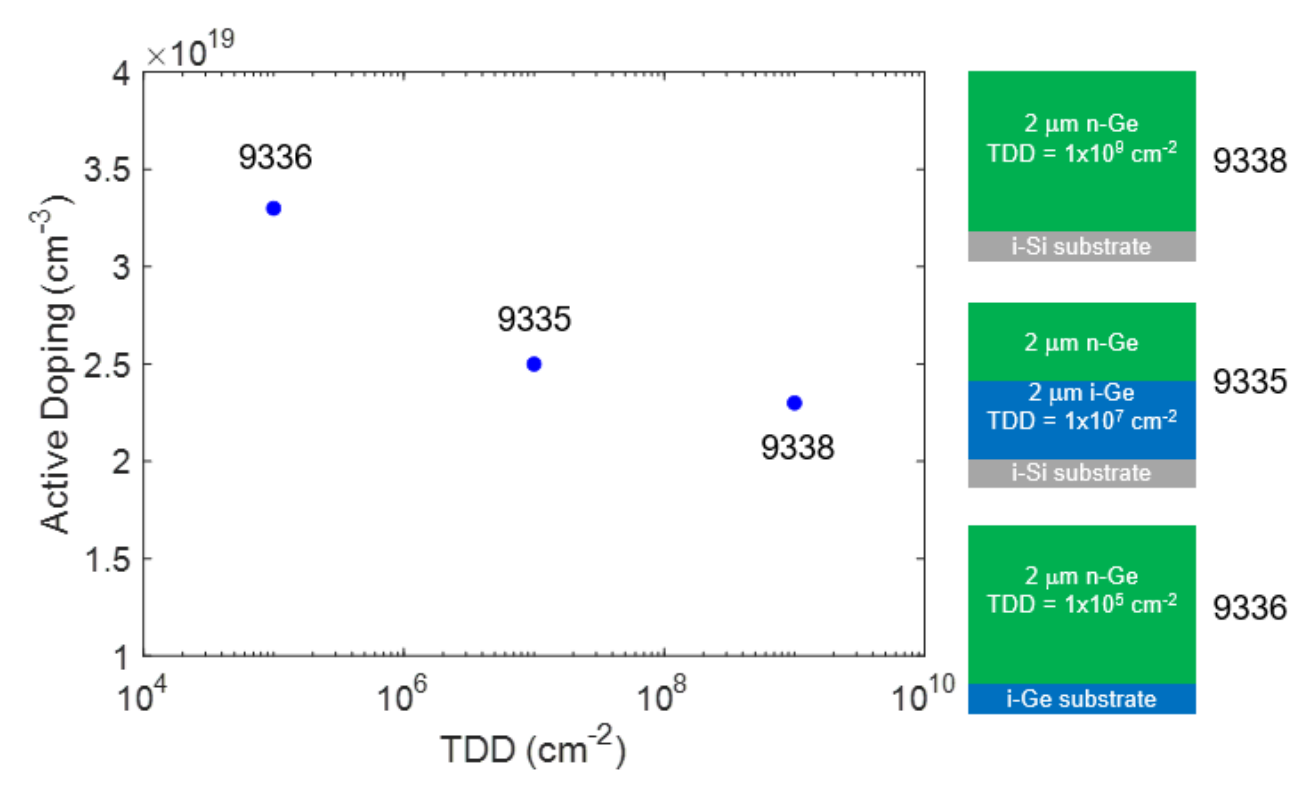

Figure 4: Active doping as a function of the TDD and schematics of the samples 9338, 9335 and 9336.

As it can be seen from the plot, a lower TDD leads to an increased active doping level. Nevertheless, it should be pointed out that the activation levels are still far from $100 \%$ and that the formation of vacancy-donor complexes [35] remains the main factor limiting full dopant activation. 


\section{POST-GROWTH THERMAL TREATMENTS TO INCREASE THE DOPANT ACTIVATION}

The samples investigated in the previous section clearly demonstrate that the concentration of $\mathrm{P}$ atoms incorporated in the Ge thin films is much higher than those effectively contributing to the active doping. In this section we will investigate the possibility to increase the $\mathrm{P}$ activation by employing two post-growth thermal annealing techniques: the rapid thermal annealing (RTA) and the pulsed laser melting (PLM).

\subsection{Rapid thermal annealing}

RTA is a standard technique to promote dopant activation in semiconductors doped through ion implantation. After the implantation, dopants are trapped in interstitial positions into the crystal lattice and the RTA allows them to reach substitutional sites. In our case we will investigate the possibility to use the same technique to promote the dissolution of vacancy-donor clusters, thus enhancing the overall activation. The sample consists of a $500 \mathrm{~nm}$ thick Ge epilayer, grown at $460{ }^{\circ} \mathrm{C}$ at a rate of $1 \mathrm{~nm} / \mathrm{s}$ and with a $\mathrm{PH}_{3} / \mathrm{GeH}_{4}$ ratio of 0.035 . After the growth we performed RTA at four different maximum temperatures of $600{ }^{\circ} \mathrm{C}, 650{ }^{\circ} \mathrm{C}, 700{ }^{\circ} \mathrm{C}$, and $750{ }^{\circ} \mathrm{C}$. The maximum temperature was kept constant for 30 seconds. The chemical profiles measured by SIMS show a severe diffusion of the dopants toward the substrate and the surface. Such diffusion reduces the available $\mathrm{P}$ atoms in the Ge-on-Si layer and creates non-uniform doping profiles that are not suitable for plasmonic applications, where a box-like doping profile over a few skin depths is desirable. Interestingly the active doping levels in the samples annealed at $700{ }^{\circ} \mathrm{C}$ and $750{ }^{\circ} \mathrm{C}$ are almost matching those of the incorporated $\mathrm{P}$, thus confirming that fast thermal treatments are able to increase the dopant activation [38,39].
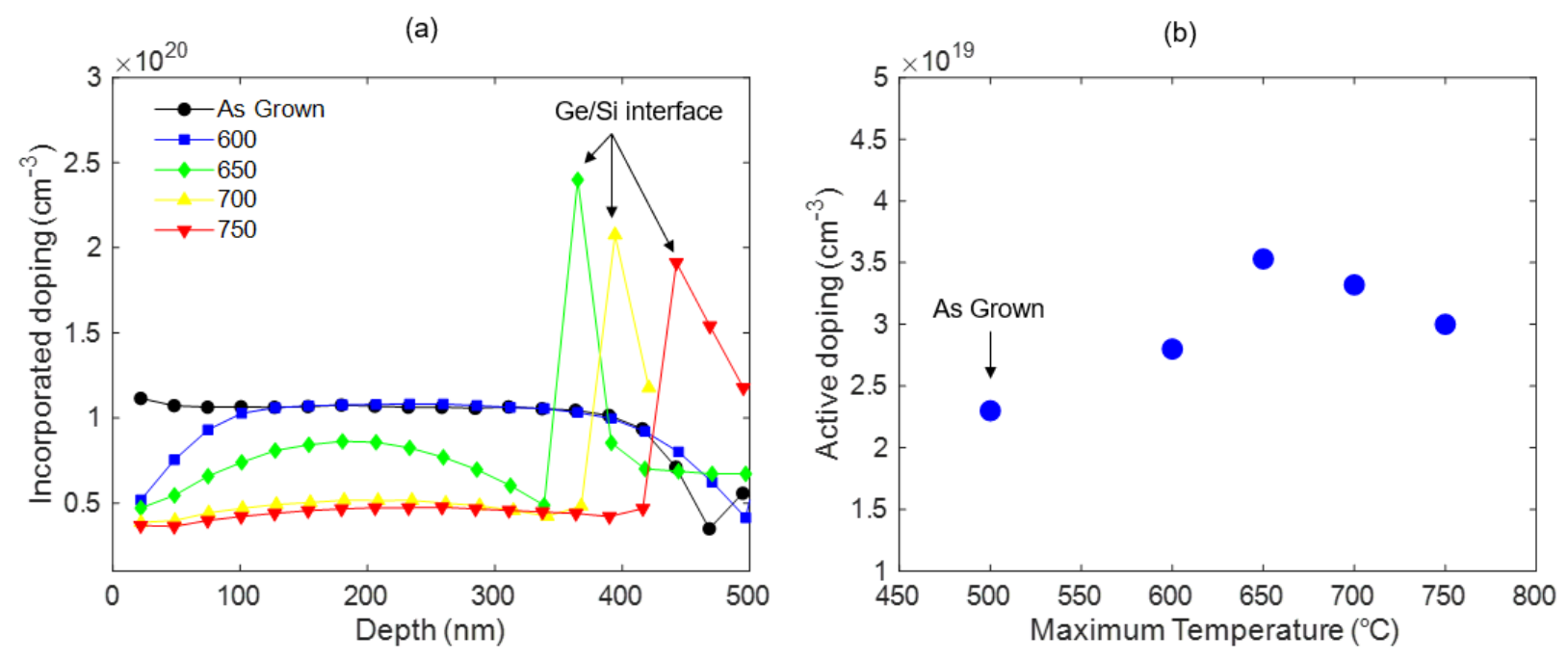

Figure 5: (a) Chemical Profile of $\mathrm{P}$ as a function of the depth as measured by SIMS; (b) Active doping as a function of the RTA maximum temperature. A dedicated sample (9547) has been grown for this experiment.

\subsection{Pulsed Laser Melting}

An alternative approach to increase the active doping concentration is pulsed laser melting (PLM). In the PLM process one or more laser pulses are used to melt the ion-implanted or in-situ doped semiconductor. The subsequent fast, out of equilibrium recrystallization process enhances the substitutional incorporation of dopants in the material. Moreover, by using pulse durations in the nanoseconds range, dopant diffusion is inhibited and a box-like doping profile can be obtained. We performed several experiments [40] on samples with different P incorporations in order to assess the potential of PLM to achieve full activation. 
Table 4. Main characteristics of samples used for PLM experiments. The Ge thickness is $500 \mathrm{~nm}$ for all the samples.

\begin{tabular}{|c|c|c|c|c|c|}
\hline Sample & $\begin{array}{c}\text { Growth } \\
\text { Temp. }\left({ }^{\circ} \mathbf{C}\right)\end{array}$ & $\begin{array}{c}\text { Pulse energy } \\
\text { density } \\
\left(\mathbf{J} / \mathbf{c m}^{-\mathbf{2}}\right)\end{array}$ & $\begin{array}{c}\text { Pulse } \\
\text { wavelength/duration } \\
(\mathbf{n m} / \mathbf{n s})\end{array}$ & $\begin{array}{c}\text { Active } \\
\mathbf{d o p i n g} \\
\left(\mathbf{c m}^{-\mathbf{3}}\right)\end{array}$ & $\begin{array}{c}\text { Incorporated } \\
\mathbf{P} \text { atoms } \\
\left(\mathbf{c m}^{-\mathbf{3}}\right)\end{array}$ \\
\hline 9547 & 460 & 1 & $308 / 28$ & $8.8 \mathrm{E}+19$ & $1.1 \mathrm{E}+20$ \\
\hline 10255 & 450 & 1.9 & $308 / 153$ & $1.3 \mathrm{E}+20$ & $2.1 \mathrm{E}+20$ \\
\hline 10258 & 350 & 0.4 & $355 / 7$ & $2.2 \mathrm{E}+20$ & $4.0 \mathrm{E}+20$ \\
\hline
\end{tabular}

As can be seen from table 4, PLM is very effective in enhancing the active doping. The maximum electron density achieved is $2.2 \times 10^{20} \mathrm{~cm}^{-3}$, which corresponds to a screened plasma frequency of $\omega^{*} \cong 3500 \mathrm{~cm}^{-1}\left(\lambda^{*} \cong 3 \mu \mathrm{m}\right)$.

(a)

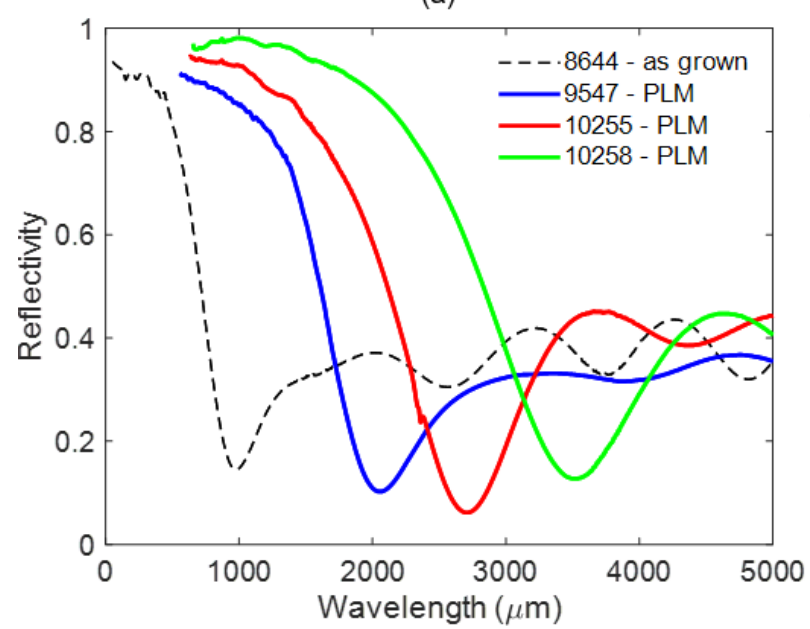

(b)

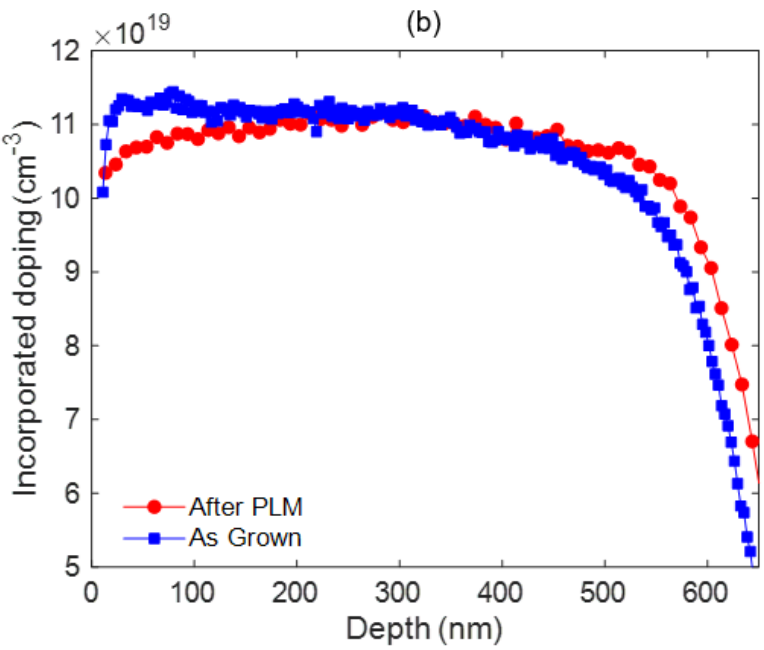

Figure 6: (a) Reflectivity as a function of the wavenumber for the three samples treated with PLM and for the reference sample 8644 (dashed line). (b) Incorporated doping as a function of the depth for sample 9547 before and after the PLM.

This result demonstrates the potential of heavily n-doped Ge-on-Si as a material platform to exploit plasmonic effects in the MIR between 3 and $15 \mu \mathrm{m}$ wavelengths. The incorporated doping profiles of sample 9547 shown in fig. $5 \mathrm{~b}$ confirms that the dopant diffusion is inhibited and a box-like profile can be obtained, even if it has to be mentioned that the active doping profile could be different from the incorporated one. 


\section{LOSSES IN GE-ON-SI THIN FILMS}

The interest toward heavily doped semiconductors for mid-infrared plasmonics has been also driven by the hypothesis that their higher DC mobility with respect to metals would result in lower losses [7]. In order to shed some light on this topic, we performed an in-depth systematic study [19] on heavily doped Ge-on-Si samples displaying different values of active doping. The dielectric function of a heavily doped semiconductor, as far as spectral ranges where interband transitions and optical phonons can be neglected, can be modelled with a Drude function:

$$
\varepsilon(\omega)=\varepsilon_{\infty}-\frac{\omega_{p}^{2}}{\omega^{2}-i \gamma \omega}
$$

where $\omega_{\mathrm{p}}$ is the unscreened plasma frequency and $\gamma$ is the scattering rate determining the losses. As a first attempt, we measured the Hall coefficient $R_{H}$ and the resistivity $\rho$ of each sample and we extracted the scattering time as:

$$
\tau_{H}=\left(\frac{m^{*}}{e}\right) R_{H} \rho
$$

By fitting the absolute reflectivity spectra of the samples with the Drude model, it is also possible to extract the Drude scattering time as:

$$
\tau_{D}=\frac{1}{2 \pi c \gamma}
$$

By looking at the data plotted in Figure 7, one can notice a significant discrepancy between $\tau_{\mathrm{H}}$ and $\tau_{\mathrm{D}}$. Indeed, the clear dependence of $\tau_{\mathrm{H}}$ on the active doping level has no counterpart in $\tau_{\mathrm{D}}$. The reason is that $\tau_{\mathrm{H}}$ is mainly determined by momentum relaxation phenomena (scattering with ionized-impurities and acoustic phonons) while $\tau_{\mathrm{D}}$ is the inelastic scattering time of oscillating electron currents, which is mainly determined by scattering with optical phonons.

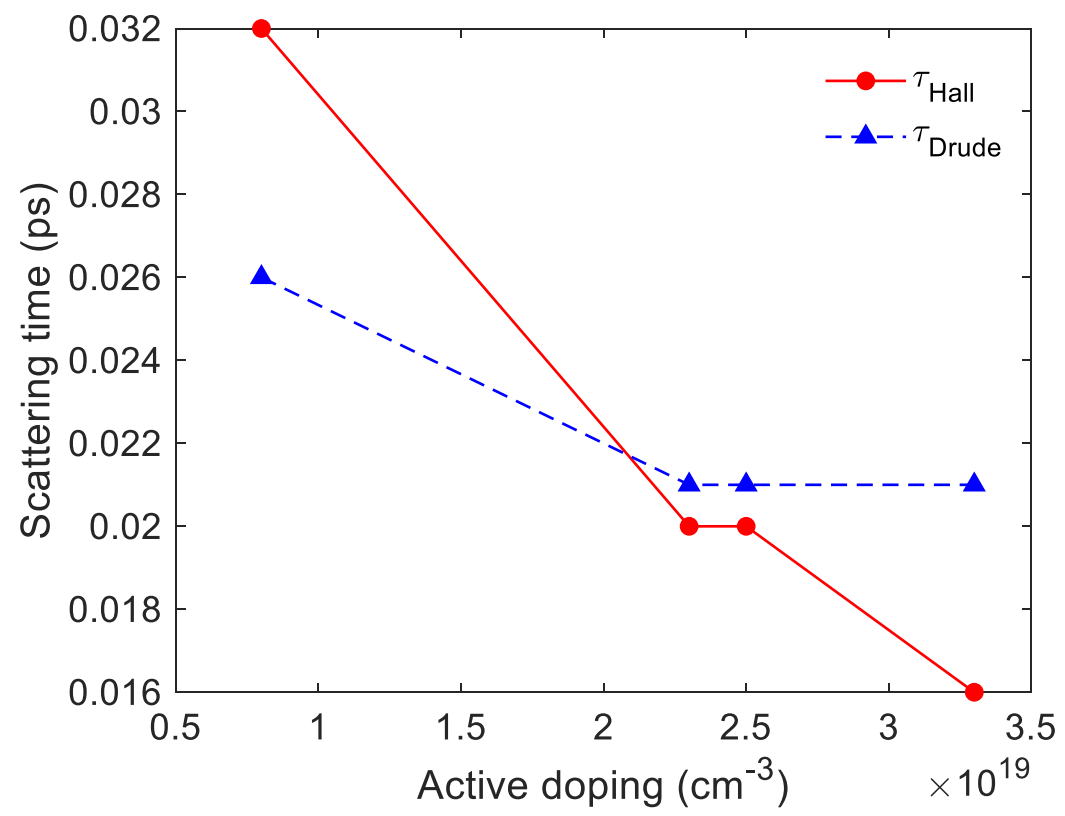

Figure 7: Scattering time as calculated from Hall measurements (red dots) and from IR reflectivity measurements ( blue triangles)

These results clearly demonstrate that the DC electrical properties of heavily doped semiconductors cannot be used to estimate the losses. By further analysing the reflectivity curves at low temperature, we realized that a single $\gamma$-value Drude 
model cannot be used to properly fit the data. For this reason, we employed Kramers-Kronig transformations [19] to extract a frequency-dependent scattering rate from the reflectivity curves. The results are shown in figure 8 . The analysis reveals that the scattering rate is strongly frequency-dependent and it mainly depends on electron-ionized impurities and electron-optical phonons scattering channels. We also found that $\gamma(\omega)$ is almost constant with doping (at least in the $10^{18}$ $10^{19} \mathrm{~cm}^{-3}$ range) and it shows a certain degree of dependence on temperature due to the phonon contribution.

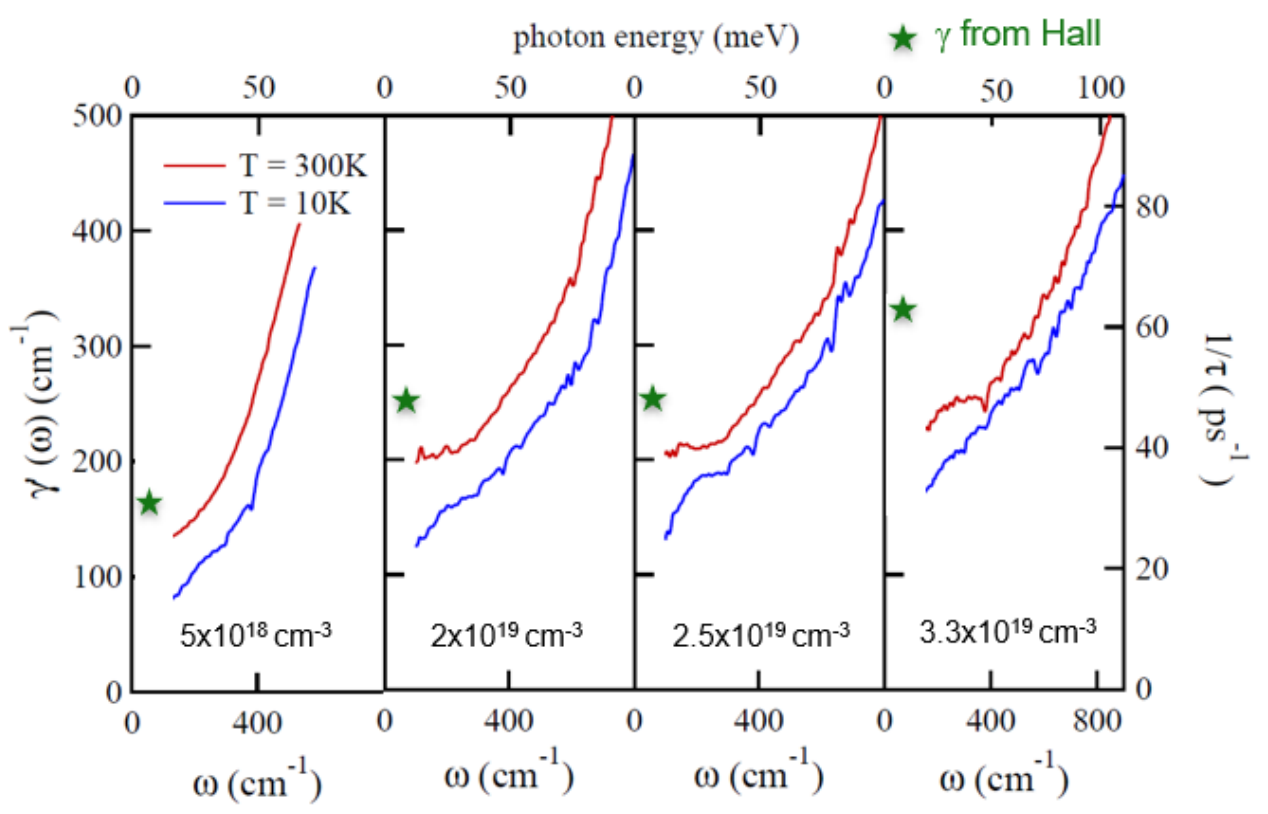

Figure 8: Experimental frequency dependent scattering rate.

\section{CONCLUSIONS}

In this work we have presented our results on the optimization of the growth protocols aiming to reach very high levels of n-type active doping in Ge thin films grown on Si. Thanks to a combination of low-temperature deposition and post-growth PLM we were able to achieve an active doping density of $N_{d} \approx 2.1 \times 10^{20} \mathrm{~cm}^{-3}$, which provides a plasmonic response down to a wavelength of about $\lambda_{\mathrm{p}}=3 \mu \mathrm{m}$. By analysing the IR reflectivity of several samples, we also demonstrated that the plasmon decay times in heavily doped semiconductors cannot be estimated by the dc mobility, nor within a DrudeLorenz model, since the electron scattering rate is strongly frequency dependent. Our work demonstrates that n-type Ge has the potential to become the material platform of choice for plasmonics exploiting materials compatible with silicon foundries.

\section{ACKNOWLEDGMENTS}

The research leading to these results has received funding from the European Union's Seventh Framework Programme under grant agreement no. 613055

\section{REFERENCES}

[1] Limaj O., D’Apuzzo F., Di Gaspare A., Giliberti V., Domenici F., Sennato S., Bordi F., Lupi S. and Ortolani M., "MidInfrared Surface Plasmon Polariton Sensors Resonant with the Vibrational Modes of Phospholipid Layers" J. Phys. Chem. C 117(37), 19119-19126 (2013).

[2] Chang Y.-C., Wagli P., Paeder V., Homsy A., Hvozdara L., van der Wal P., Di Francesco J., de Rooij N.F. Herzig H.P., "Cocaine detection by a mid-infrared waveguide integrated with a microfluidic chip" Lab Chip 12, 3020-3023 (2012). 
[3] Lin P. T., Kwok S.W., Lin H. Y. G., Singh V., Kimerling L.C., Whitesides G.M. and Agarwal A.. "Mid-Infrared Spectrometer Using Opto-Nanofluidic Slot-Waveguide for Label-Free On-Chip Chemical Sensing" Nano Lett. 14(1), 231238(2014).

[4] Adato R. and Altug H. "In-situ ultra-sensitive infrared absorption spectroscopy of biomolecule interactions in real time with plasmonic nanoantennas" Nat. Comm. 4, 2154 (2013).

[5] Galli I., Bartalini S., Borri S., Cancio P., Mazzotti D., De Natale P., Giusfredi G., "Molecular gas sensing below parts per trillion: radiocarbon-dioxide optical detection" Phys Rev Lett. 107, 270802 (2011).

[6] S.A. Maier, Brongersma M.L., Kik P.G., Meltzer S., A.A.G. Requicha, Atawater H.A., "Plasmonics-A route to Nanoscale Optical Devices" Adv. Mater. 13(19), 1501-1505 (2001)

[7] Boltasseva A., "Low -Loss Plamonic Metamaterials" Science 331(6015), 290-291 (2011)

[8] Naik G.V., Shalaev V.M., and Boltasseva A., "Alternative plamonic materials: beyond gold and silver" Adv. Mater. 25(24), 3264-3294 (2013).

[9] Hoffman A. J., Alekseyev L., Howard S. S.,Franz K. J., Wasserman D., Podolskiy V.A., Narimanov E.E., Sivco D.L.,Gmachl D., "Negative refraction in semiconductor metamaterials" Nat. Mater. 6(12), 946-950 (2007).

[10] Ginn J.C., Jarecki R.L., Shaner E.A., Davids P.S., "Infrared plasmons on heavily doped silicon” J. Appl.Phys. 110(4), 043110 (2011).

[11] Shahzad M., Medhi G., Peale R.E., Buchwald W.R., Cleary J.W., Soref R., Boreman G.D., Edwards O.," Infrared surface plasmons on heavily doped silicon” J. Appl. Phys. 110(12), 123105 (2011).

[12] Li D., Ning C.Z., "All-semiconductor active plasmonic system in mid-infrared wavelengths" Opt. Express 19 (15), 14594 (2011).

[13] Law S., Adams D.C., Taylor A.M., Wasserman D., “Mid-infrared designer metals” Opt. Express 20(11), 12155-12165 (2012).

[14] Law S., Yu L., Rosenberg A., Wasserman D., "All-Semiconductor Plasmonic Nanoantennas for Infrared Sensing" Nano Lett. 13(9), 4569-4574 (2013).

[15] Baldassarre L., Sakat E., Frigerio J., Samarelli A., Gallacher K., Calandrini E., Isella G., Paul D.J., Ortolani M., Biagioni P., "Midinfrared Plasmon-Enhanced Spectroscopy with Germanium Antennas on Silicon Substrates" Nano Lett. 15(11), 7225-7231 (2015).

[16] A. Berrier, Ulbricht R., Bonn M., Rivas J.G.,"Ultrafast active control of localized surface plasmon resonances in silicon bowtie antennas" Opt. Express 18(22), 23226-23235 (2010).

[17] Wagner M., McLeod A.S., Maddox S.J.,Fei Z.,Liu M., Averitt R.D., Fogler M.M., Bank S.R., Keilmann F., Basov D.N., "Ultrafast Dynamics of Surface plasmons in InAs by time resolved infrared nanospectroscopy" Nano Lett. 14(8), 4529-4534 (2014).

[18] Fischer M.P., Schmidt C., Sakat E., Stock J., Samarelli A., Frigerio J., Ortolani M.., Paul D.J., Isella G., Leitenstorfer A., Biagioni P., Brida D., "Optical activation of germanium plasmonic antennas in the mid-infrared" Phys. Rev. Lett. 117(4), 047401 (2016).

[19] Frigerio J., Ballabio A., Isella G., Sakat E., Pellegrini G., Biagioni P., Bollani M., Napolitani E., Manganelli C., Virgilio M., Grupp A., Fischer M.P., Brida D., Gallacher K., Paul D.J., Baldassarre L., Calvani P., Giliberti V., Nucara A., Ortolani M., "Tunability of the dielectric function of heavily doped germanium thin films for mid-infrared plasmonics" Phys. Rev. B 94(8), 085202 (2016).

[20] Taliercio T., Biagioni P., "Semiconductor infrared plasmonics" Nanophotonics 8(6), 949-990 (2019).

[21] Mashanovich G.Z., Mitchell C.J.,Soler Penades J., Khokhar A.Z., Littlejohns C.G., Cao W., Qu Z., Stanković S., Gardes F.Y., Masaud T.B., Chong H., Mittal V., Murugan G.S., Wilkinson J.S., Peacock A.C., Nedeljkovic M.," Germanium mid-infrared photonic devices" J. Light. Tech. 35(4), 624-630 (2016).

[22] Yang M., Guo Y., Wang J., Han Z., Wada K., Kimerling L.C., Agarwal A.M., Michel J., Li G., Zhang L., "Mid-IR supercontinuum generated in low-dispersion Ge-on-Si waveguides pumped by sub-ps pulses" Opt. Express 25(14), 1611616122 (2107).

[23] Griskeviciute U., Millar R.W., Gallacher K., Valente J., Paul D.J.," Ge-on-Si waveguides for sensing in the molecular fingerprint regime", Opt. Express 28(4), 5749-5757 (2020).

[24] Gallacher K., Millar R.W.,Paul D.J., Frigerio J., Ballabio A., Isella G., Rusconi F., Biagioni P., Giliberti V., Sorgi A., Baldassarre L., Ortolani M., " Characterization of integrated waveguides by atomic-force-microscopy-assisted midinfrared imaging and spectroscopy" Opt. Express 28(15), 22186-22199 (2020).

[25] J.-M. Ramirez, Q. Liu, V. Vakarin, J. Frigerio, A. Ballabio, X. Le Roux, D. Bouville, L. Vivien, G. Isella, and D. Marris-Morini, "Graded SiGe waveguides with broadband low-loss propagation in the mid infrared," Opt. Express 26(2), 870-877 (2018). 
[26] V. Vakarin, J.-M. Ramírez, J. Frigerio, A. Ballabio, X. Le Roux, Q. Liu, D. Bouville, L. Vivien, G. Isella, and D. Marris-Morini, "Ultra-wideband Ge-rich silicon germanium integrated Mach-Zehnder interferometer for midinfrared spectroscopy," Opt. Lett. 42(17), 3482-3485 (2017).

[27] J. M. Ramírez, Q. Liu, V. Vakarin, X. L. Roux, J. Frigerio, A. Ballabio, C. Alonso-Ramos, E. Simola, L. Vivien, G. Isella, and D. Marris-Morini, "Broadband integrated racetrack ring resonators for long-wave infrared photonics," Opt. Lett. 44(2), 407 (2019).

[28] Q. Liu, J. M. Ramírez, V. Vakarin, X. L. Roux, C. Alonso-Ramos, J. Frigerio, A. Ballabio, E. Simola, D. Bouville, L. Vivien, G. Isella, and D. Marris-Morini, "Integrated broadband dual-polarization Ge-rich SiGe mid-infrared Fouriertransform spectrometer," Opt. Lett. 43(20), 5021 (2018).

[29] Sinobad M., Monat C., Luther-Davies B., Ma P., Madden S., J. Moss D., Mitchell A., Allioux D., Orobtchouk R., Boutami S., Hartmann J.-M., Fedeli J.M., Grillet C., "Mid-infrared octave spanning supercontinuum generation to $8.5 \mu \mathrm{m}$ in silicon-germanium waveguides" Optica 5(4), 360-366 (2018).

[30] Montesinos-Ballester M., Lafforgue C., Frigerio J., Ballabio A., Vakarin V., Liu Q., Ramirez J.M., Le Roux X., Bouville D., Barzaghi A., Alonso-Ramos C., Vivien L., Isella G. Marris-Morini D., "On-chip supercontinuum generation from 3 to $13 \mu \mathrm{m}$ wavelength" ACS Photonics 7(12) 3423-3429 (2020).

[31] Montesinos-Ballester M., Vakarin V., Liu Q., Le Roux X., Frigerio J., Ballabio A., Barzaghi A., Alonso-Ramos C., Vivien L., Isella G. Marris-Morini D., "Ge-rich graded SiGe waveguides and interferometers from 5 to $11 \mu \mathrm{m}$ wavelength range" Opt. Express 28(9), 12771-12779 (2020).

[32] Montesinos-Ballester M., Vakarin V., Ramirez J.M., Liu Q., Alonso-Ramos C., Le Roux X., Frigerio J., Ballabio A., Barzaghi A., Deniel L., Bouville, D., Laurent V., Isella G., Marris-Morini D., "Optical modulation in Ge-rich SiGe waveguides in the mid-IR wavelength range up to $11 \mu \mathrm{m}$ " Comm. Mat. 1(1), 1-6 (2020).

[33] Samarelli A., Frigerio J., Sakat E., Baldassarre L. Gallacher K., Finazzi M., Isella G., Ortolani M., Biagioni P., Paul D.J., "Fabrication of mid-infrared plasmonic antennas based on heavily doped germanium thin films" Thin Solid Films 602, 52-55 (2016).

[34] Höhler H., Atodiresei N., Schroeder K., Zeller R., Dederichs P. H.,"Vacancy complexes with oversized impurities in Si and Ge" Phys. Rev. B 71(3) 035212 (2005).

[35] Prucnal S., Liedke M.O., Wang X., Butterling M., Posselt M., Knoch J., Windgassen H., Hirschmann E., Berencen Y., Rebhole L., Wang M., Napolitani E., Frigerio J., Ballabio A., Isella G., Hubner R., Wagner A., Bracht H., Helm M., Zhou S., "Dissolution of donor-vacancy clusters in heavily doped n-type germanium" New. J. Phys. 22(12), 123036 (2020). [36] Read W.T., "Theory of dislocations in germanium", The London, Edinburgh and Dublin Philosophical Magazine and Journal of Science 45(367), 775-796 (1954).

[37] Geiger R., Frigerio J., Suess M.J., Chrastina D., Isella G., Spolenak R., Faist J., Sigg H., "Excess carrier lifetimes in Ge layers on Si" Appl. Phys. Lett. 104(6) 062106 (2014).

[38] Shim J., Song I., Jung W.S., Nam J., Leem J.W., Yu J.S., Kim D.E., Cho W.J., Yun D.H., Heo J., Park W., Park JinHong, Sarasawat K.C., "Effects of thermal annealing on in situ phosphorous doped germanium n+/p junction" IEEE Elec. Dev. Lett. 34(1), 15-17 (2013).

[39] Prucnal S., Frigerio J., Napolitani E., Ballabio A., Berencén Y., Rebohle L., Wang M., Böttger R., Voelskow M., Isella G., Hübner R., Helm M., Zhou S., Skorupa W.,'In situ ohmic contact formation for n-type Ge via non equilibrium processing" Semicon. Sci. Tech. 32(11) 115006 (2017).

[40] Frigerio J., Ballabio A., Gallacher K., Giliberti V., Baldassarre L., Millar R., Milazzo R., Maiolo L., Minotti A., Bottegoni F., Biagioni P., Paul D.J., Ortolani M., Pecora A., Napolitani E., Isella G., "Optical properties of highly n-doped germanium obtained by in situ doping and laser annaeling” J. Phys. D 50(46), 465103 (2017). 